\title{
Investigation of Amplification Parameters in Q-switched Fiber Oscillator-Amplifier Systems
}

\author{
Narges Shafii Mousavi ${ }^{\mathrm{a}}$, Parviz Parvin ${ }^{\mathrm{a}}$, and Maryam Ilchi-Ghazaani ${ }^{\mathrm{b}}$

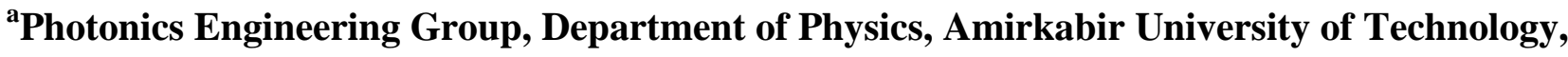 \\ Tehran, Iran \\ ${ }^{b}$ Photonics and Quantum Technologies Research School, Nuclear Science and Technology \\ Research Institute (NSTRI), Tehran, Iran \\ *Corresponding author: parvin@aut.ac.ir
}

Regular paper: Received: Oct. 23, 2020, Revised: Dec. 13, 2020, Accepted: Dec. 23, 2020, Available Online: Dec. 25, 2020, DOI: 10.29252/ijop.14.2.155

\begin{abstract}
In this paper, an analytical model is presented to compare the monolithic endpumped and distributed side-pumped arrangements in the master oscillator power amplifier (MOPA) of Q-switched (QSW) double-clad (DC) ytterbium (Yb)-doped fiber system. First, the time-dependent rate equations are solved numerically by the finite difference method and the output pulse characteristics are obtained. For more amplifying, the laser pulse is injected into the amplifier and the gain and saturation coefficients are obtained by using the best fitting between the outcoming data from solving rate equations and the transient amplification relation, based on the least squares method (LSM). Finally, the dependence of pump power and dopant concentration on the cavity amplifying parameters are investigated.
\end{abstract}

KEYWORDS: Gain coefficient; Saturation power; End-pumped; Side-pumped; distributed; Q-switched; Fiber laser; Masteroscillator power-amplifier.

\section{INTRODUCTION}

In the design of fiber lasers and amplifiers, gain and saturation coefficients are considered as the main parameters [1], [2]. Among different gain mediums, the rare-earth ions of ytterbium $(\mathrm{Yb})$ are very useful and considerable in high power fiber lasers especially in pulsed mode [3], [4].
In addition, QSW fiber lasers are highly applicable in small areas, material processing, laser ranging, medicine, remote sensing, laser surgery, welding and radiation [5-9].

The most popular power scaling way of achieving high peak powers is the MOPA configuration which, consists of a master oscillator (MO) and single or multi-stages power amplifier (PA). The MO generates a low power coherent beam, whereas the PA can elevate the output pulse several folds [10]. In this regard, modeling is a useful method for optimizing parameters before the experiment. In fact, the simulation of a QSW fiber laser performs in two main ways: a point model [11] and a traveling wave scheme [12].

Moreover, the amplifier is characterized by a couple of significant coefficients i.e., smallsignal gain $\left(\gamma_{0}(z)\right)$ and saturation power $\left(P_{\text {sat }}\right)$ [1], [13].

Here, a series of systematic theoretical analysis is carried out to determine the amplifying parameters in MOPA. Several papers have been focused on numerical modeling and experimental measurement of those significant optical elements in QSW fiber lasers [4].

In order to obtain high output power, the pumping technology is generally divided into 
two categories: end-pumped [14] and sidepumped [15].

The principle of end-pumped mode is the simplest technology, where the output light from a laser diode module is coupled by using the optical system [16], [15]. For side-pumped method, the V-groove technology is the most common technology [17], [18].

In this paper, modeling of a typical endpumping and a distributed array of a QSW DC Yb:silica fiber laser amplifier is performed. Here, the theoretical model for a couple of main pumping technologies in MOPA layout is represented as follows:

- single-stage monolithic end-pumping configuration

- two-stage distributed side-pumped array

Then, the effect of dopant concentration and input pump power parameters on small-signal gain and amplifier saturation power is investigated.

\section{THEORY}

The schematic illustration of a typical QSW DC Yb:silica fiber laser amplifier under the (a) end-pumping and (b) distributed side-pumping arrays is shown in Fig. 1. The length of the amplifier at end-pumping is equal to $L$, however, the distributed array includes two amplifiers, each with a length of $L / 2$.

A fiber Bragg grating (FBG) with high reflectance (HR) is used to reflect the first order beam. Another FBG with low reflectance (LR) is employed at the end of the oscillator as an output coupler (OC). Hence, the oscillator is characterized by a couple of FBGs.

The simplified time-dependent rate equations are given by [19], [20]:

$N=N_{1}+N_{2}$

$$
\begin{aligned}
& \frac{\partial N_{2}(z, t)}{\partial t}+\frac{N_{2}(z, t)}{\tau}= \\
& \frac{\Gamma_{p} \lambda_{p}}{h c A}\left[\sigma_{a p}\left(\lambda_{p}\right) N_{1}(z, t)-\sigma_{e p}\left(\lambda_{p}\right) N_{2}(z, t)\right] \times \\
& \times p_{p}(z, t)+ \\
& +\frac{\Gamma_{s} \lambda_{s}}{h c A}\left[\sigma_{a s}\left(\lambda_{s}\right) N_{1}(z, t)-\sigma_{e s}\left(\lambda_{s}\right) N_{2}(z, t)\right] \\
& \left(p_{s}^{+}(z, t)+p_{s}^{-}(z, t)\right) \\
& \frac{\partial p_{p}(z, t)}{\partial z}+\frac{1}{v_{p}} \frac{\partial p_{p}(z, t)}{\partial t}=\Gamma_{p}\left[\sigma_{e p}\left(\lambda_{p}\right) N_{2}(z, t)-\right. \\
& \left.\sigma_{a p}\left(\lambda_{p}\right) N_{1}(z, t)\right] p_{p}(z, t)-\alpha\left(\lambda_{p}\right) p_{p}(z, t) \\
& \pm \frac{\partial p_{s}^{ \pm}(z, t)}{\partial z}+\frac{1}{v_{s}} \frac{\partial p_{s}^{ \pm}(z, t)}{\partial t}=\Gamma_{s}\left[\sigma_{e s}\left(\lambda_{s}\right) N_{2}(z, t)-\right. \\
& \left.-\sigma_{a s}\left(\lambda_{s}\right) N_{1}(z, t)\right] p_{s}^{ \pm}(z, t)+S \alpha_{R S} p_{s}^{\mp}(z, t)- \\
& \alpha\left(\lambda_{s}\right) p_{s}^{ \pm}(z, t)+2 \sigma_{e s}\left(\lambda_{s}\right) N_{2}(z, t) \frac{h c^{2}}{\lambda_{s}^{3}} \Delta \lambda_{s}
\end{aligned}
$$

where, $N$ denotes the dopant volume density, $N_{1}$ and $N_{2}$ are ground and upper level populations, respectively and $p_{s}^{\mp}$ is the signal power that propagates along positive and negative $z$ direction in the core. $P_{p}$ ascertains the pump power and $v_{p}$ and $v_{s}$ represent the group velocities of the pump and signal in the fiber, $c$ is the light velocity in the vacuum, $\tau$ is the fluorescence lifetime and $\mathrm{A}$ is the doped area of the fiber. Furthermore, $\sigma_{a p}\left(\sigma_{e p}\right)$ and $\sigma_{a s}\left(\sigma_{e s}\right)$ denote the absorption (emission) cross sections of $\mathrm{Yb}$ for pump and signal, accordingly. In addition, $\alpha_{\mathrm{P}}\left(\alpha_{\mathrm{S}}\right)$ indicates the fiber attenuation of the pump (signal) and $\Gamma_{p}$ $\left(\Gamma_{s}\right)$ denotes the overlapping factor between the pump (signal) and the doped area of the fiber. Moreover, $\alpha_{R S}$ is the Rayleigh-scattering coefficient, and corresponding $S$ refers to the attenuation coefficient.

The boundary conditions of partial differential rate equations (1)-(4) are given as below [19]: 
$p_{p}^{+}(0, t)=\eta_{p} p_{p 1}$

$p_{s}^{+}(0, t)=p_{s}^{-}(0, t)\left[R_{H R}\left(\lambda_{s}\right) T_{1}^{2}(t) \eta_{1}^{2} \eta_{c p l}^{2}+R_{r e s}\right](6)$

$p_{s}^{+}(L, t)=p_{s}^{+}(L, t) R_{O C}\left(\lambda_{s}\right) \eta_{2}^{2}$

where, $R_{H R}$ and $R_{O C}$ are the reflectances of the HR mirror and the OC-FBG, respectively. Furthermore, $\eta_{p} \quad\left(\eta_{c p l}\right)$ is the coupling efficiency (signal transmittance) of the pump coupler, $\eta_{1}$ is related to the signal pass loss resulting from optical coupling and component' losses, $\eta_{2}$ ascertains the splicing loss between the $\mathrm{Yb}$ - doped $\mathrm{DC}$ fiber and the FBGs, $R_{\text {res }}$ stands for the residual facet reflectivity and $p_{p 1}$ is a constant pump power. According to AOM temporal response, $T_{1}(t)$ and $T_{0}(t)$ are on / off switching operational durations, respectively. When the AOM element is off, the pump leads to an increase of the populated inversion. Just at the onset of the triggering, the QSW pulse is built up [13], [19].

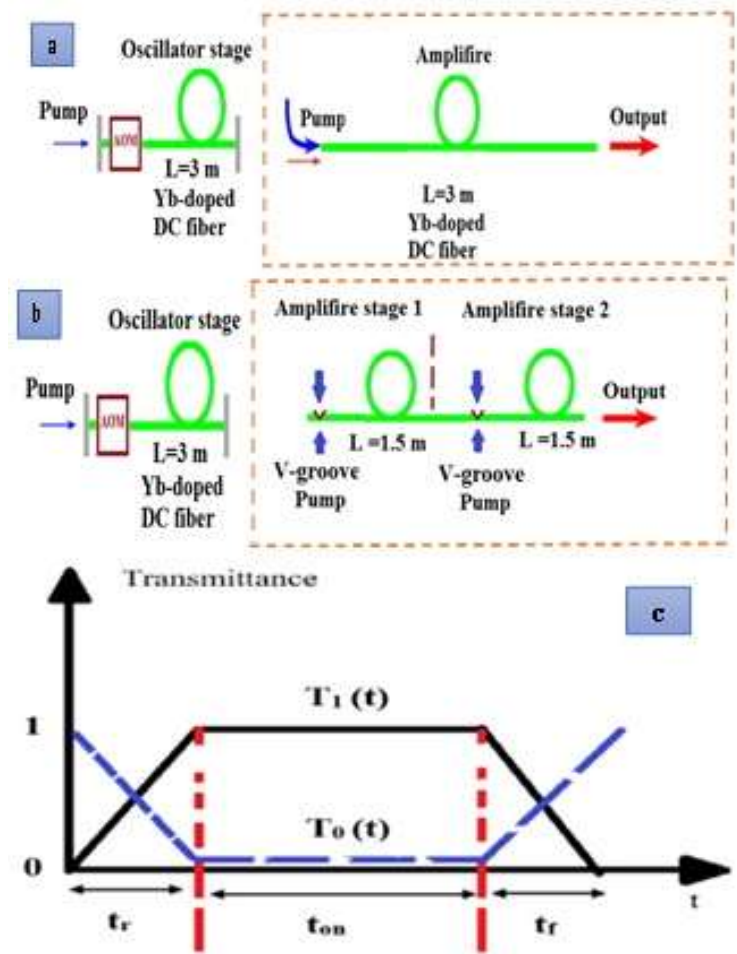

Fig. 1. Schematic of high power QSW MOPA DC fiber systems, a) end-pumping, b) distributed pumping, c) temporal transmittance of AOM including rise time $\left(\mathrm{t}_{\mathrm{r}}\right)$, opening time $\left(\mathrm{t}_{\mathrm{on}}\right)$ and fall time $\left(\mathrm{t}_{\mathrm{f}}\right)$. Note that $\mathrm{T}_{1}(\mathrm{t})$ and $\mathrm{T}_{0}(\mathrm{t})$ are on/off switching times, respectively.

In the same way, the amplifier section obeys the same Eqs. (1)-(4), where no FBG is utilized, such that $P_{S}^{-}(z, t)=0$ and the boundary condition is written as follows:

$p_{s}^{+}(0, t)=p_{\text {out }}(0, t) \times \eta$

Here, $p_{\text {out }}$ represents the output power of the oscillator and $\eta$ is the coupling losses.

The transient amplification relation is given by Frantz-Nodvik equation [21] as below:

$p_{\text {out }}=p_{\text {sat }} \ln \left\{1+\left[\exp \left(\frac{p_{\text {in }}}{p_{\text {out }}}\right)-1\right] \exp \left(\gamma_{0} L\right)\right\}$

where, $p_{\text {out }}\left(p_{\text {in }}\right)$ denotes the output (input) signal power, $\mathrm{L}$ indicates the gain length of the amplifier, $\gamma_{0}$ is the cavity gain and $p_{\text {sat }}$ shows the saturation power.

On the other hand, in the case of DC fibers, the small-signal gain can be approximated by [2] , [13]:

$$
\begin{aligned}
& \gamma_{0}(z)=\left(\frac{\lambda_{p}}{\lambda_{s}}\right)\left(\frac{1}{L}\right)\left[1-\frac{\alpha_{p}}{\alpha}\right]\left[1-e^{-\alpha L}\right]\left(\frac{p_{p}(0)}{p_{s a t}}\right) \\
& -N \Gamma_{s} \sigma_{a s}
\end{aligned}
$$

where, $p_{p}(0)$ ascertains the pump power at $z=0$, and $\alpha$ indicates total attenuation, such that $\alpha=\alpha_{p}+N \Gamma_{p} \sigma_{a p}$

In addition, the saturation parameter can be approximated by [2]:

$$
p_{\text {sat }} \cong \frac{h v_{s} A}{\left(\sigma_{a s}+\sigma_{e s}\right) \tau}\left[\frac{p_{p} \exp (-\alpha L)}{p^{\text {core }}}\right]
$$

where, $P_{\text {core }}$ is defined as the power confined in the core of fiber. 


\section{RESULTS AND DISCUSSION}

Equations (1)-(4) can be numerically solved by using the finite-difference method of MATLAB software. Table 1 shows the values of the parameters of interest.

Table 1. Parameters are used in the modeling based on rate equations

\begin{tabular}{cc}
\hline \hline Parameter & Value \\
\hline$\lambda_{\mathrm{s}}$ & $1080 \times 10^{-9} \mathrm{~m}$ \\
$\lambda_{\mathrm{P}}$ & $915 \times 10^{-9} \mathrm{~m}$ \\
$\mathrm{~A}$ & $2.83 \times 10^{-11} \mathrm{~m}^{2}$ \\
$\mathrm{~L}$ & $3 \mathrm{~m}$ \\
$\sigma_{\mathrm{as}}$ & $4.86 \times 10^{-29} \mathrm{~m}^{2}$ \\
$\sigma_{\mathrm{es}}$ & $1.99 \times 10^{-25} \mathrm{~m}^{2}$ \\
$\sigma_{\mathrm{ap}}$ & $6.7 \times 10^{-25} \mathrm{~m}^{2}$ \\
$\sigma_{\mathrm{ep}}$ & $4.2 \times 10^{-26} \mathrm{~m}^{2}$ \\
$\tau$ & $1.3 \times 10^{-3} \mathrm{~s}$ \\
$\mathrm{R}_{\mathrm{HR}}$ & $99.99 \%$ \\
$\mathrm{R}_{\mathrm{oc}}$ & $4 \%$ \\
$\mathrm{P} . \mathrm{R} . \mathrm{R}$ & $20 \mathrm{KHz}$ \\
$\eta_{1}$ & 0.8 \\
$\eta_{\mathrm{p}}$ & 0.85 \\
$\eta_{2}$ & 0.98 \\
$\mathrm{R}_{\mathrm{res}}$ & $10^{-5}$ \\
$\Gamma_{\mathrm{p}}$ & 0.0023 \\
$\Gamma_{\mathrm{s}}$ & 0.8
\end{tabular}

The gain and saturation parameters in the PA are determined by using the neutral density filters placed before the amplifier entrance. Afterward, the input signal power inserted to the amplifier is varied by changing the pump power of the oscillator stage and the amplified signal of MOPA is subsequently obtained for various pump powers fed into the amplifier. Generally, Fig. 2 plots the signal output powers in terms of input powers at several injected pump powers for distinguished pumping configurations including the singlestage monolithic end-pumping scheme and two-stage distributed side-pumping array.

The output signal increases nonlinearly against input signal power and obviously elevates at higher pump powers, which is due to the nonuniformity of pump power. The scattered data emphasizes the input/output signals extracted from the numerical simulation, while the solid curves attest to the Frantz-Nodvik analytical amplification relation given by Eq. (9). In accordance with the best fitting of the scattered data by means of the LSM, the $\gamma_{0}(z)$ and $P_{\text {sat }}$ values are determined. Note that, we need a wide range of input pump power (0$0.03 \mathrm{~W})$ to obtain small-signal gain and saturation parameters. Particularly, the small input powers are essential to determine the small-signal gain accurately.

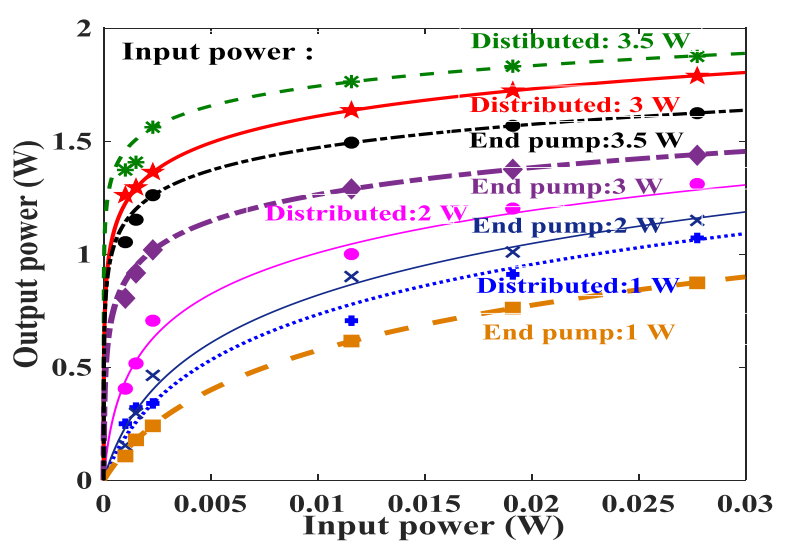

Fig. 2. Amplifier output peak power end-pumping and distributed array versus input signal power for several incident pump powers.

Furthermore, Figs. 3 and 4 depict the $\operatorname{Gain}(\mathrm{dB})=10 \times \log \left(\frac{P_{\text {out }}}{P_{\text {in }}}\right), \quad$ in terms of input/output signal powers for several pumping modes which correspond to the data given in Fig. 2 for end-pumping and distributed arrays. The highest gain is obtained for the distributed array at $3.5 \mathrm{~W}$ in comparison of end-pumping because of the impact of side-pumping injection at $L / 2$.

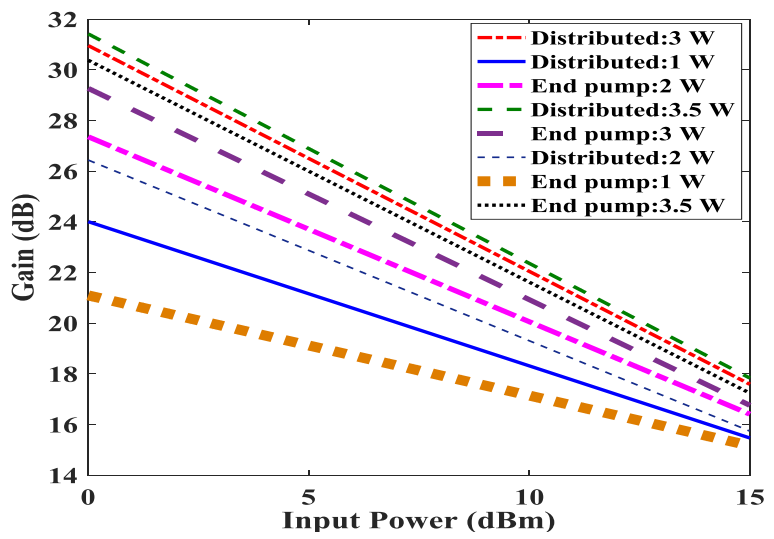

Fig. 3. The gain versus input signal of end-pumping and distributed array in PA versus different pump powers. 


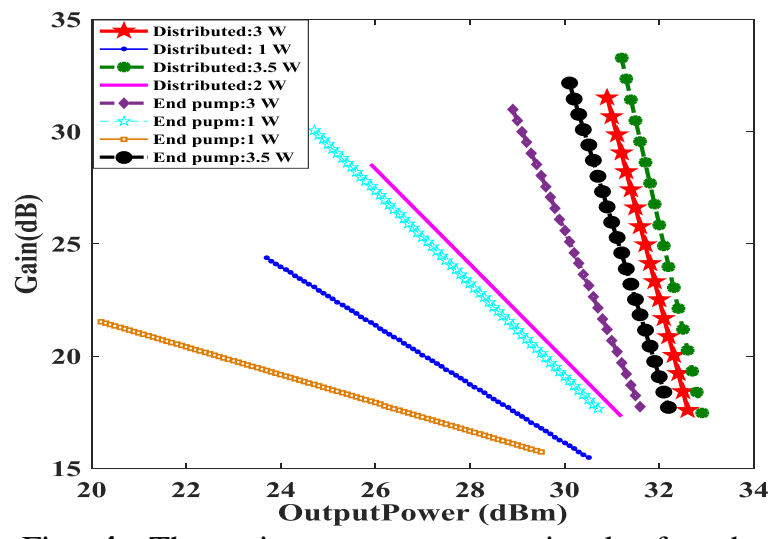

Fig. 4. The gain versus output signal of endpumping and distributed array in PA versus different pump powers.

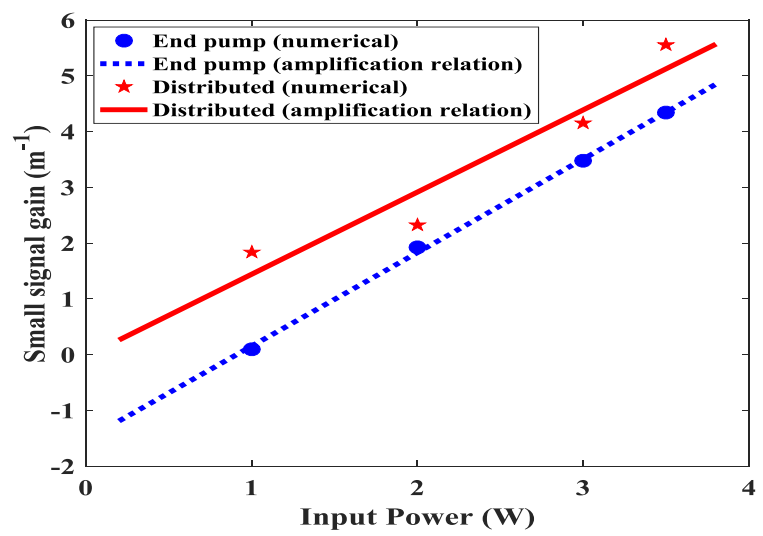

Fig. 5. Small-signal gain of end-pumping and distributed array versus pump power at a fixed $3.35 \times 10^{26} \mathrm{~m}^{-3}$ concentration.

In the following, the effect of pump power and $\mathrm{Yb}$ dopant concentration on amplifying coefficients is investigated. At first, the variation of $\gamma_{0}(z)$ and Psat in terms of input pump powers in PA stage for the end-pumping and distributed side-pumping distributions are shown in Figs. 5 and 6, respectively. The gain coefficient and the saturation power increase linearly regarding the injected pump power fed into the amplifier which are in accordance with Eqs. (10) and (11). For a typical $3.5 \mathrm{~W}$ pump power at the end-pumping configuration, $\gamma_{0}(z)$ and Psat are determined to be $4.36 \mathrm{~m}-1$ and $0.3227 \mathrm{~W}$, respectively. On the other hand, at distributed mode for a typical $3.5 \mathrm{~W}$ pump power, $\gamma_{0}(z)$ and Psat are determined to be $5.5 \mathrm{~m}^{-1}$ and $0.3431 \mathrm{~W}$, respectively. In fact, the linear behavior of Psat is due to the nature of PA, which refers to the amount of energy that can be extracted from a saturation mode.

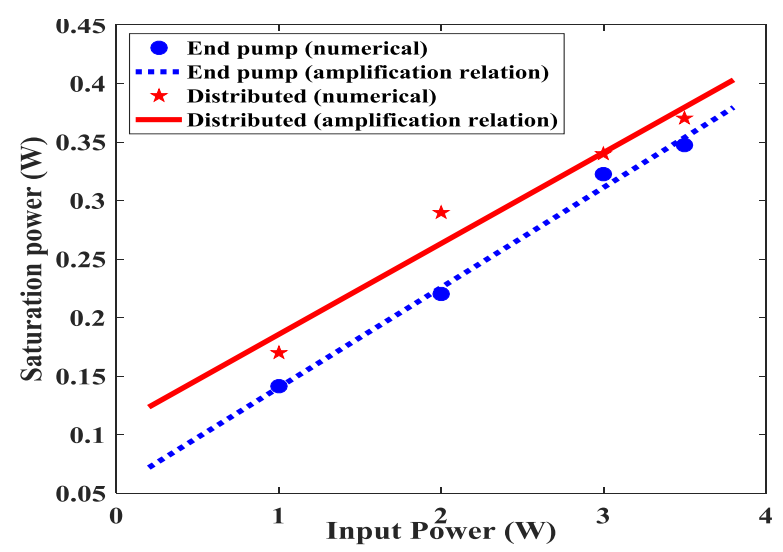

Fig. 6. Saturation power of end-pumping and distributed array versus pump power at a fixed $3.35 \times 10^{26} \mathrm{~m}^{-3}$ concentration.

In addition, $\gamma_{0}(z)$ and Psat coefficients are obtained as a function of $\mathrm{Yb}$ dopant concentration, at a certain pump power $3.5 \mathrm{~W}$. Fig. 7 depicts small-signal gain in terms of dopant concentration for various cases of onestage monolithic end-pumping and two-stage distributed side-pumping arrays of the amplifier that emphasizes a non-linear growth according to the Eq. (10). In contrast, Fig. 8 shows the exponential decay of saturation power versus dopant concentration which is in agreement with Eq. (11). The nonlinear distribution of saturation power is related to the nonlinear nature of the population inversion and the pump/signal overlapping factors.

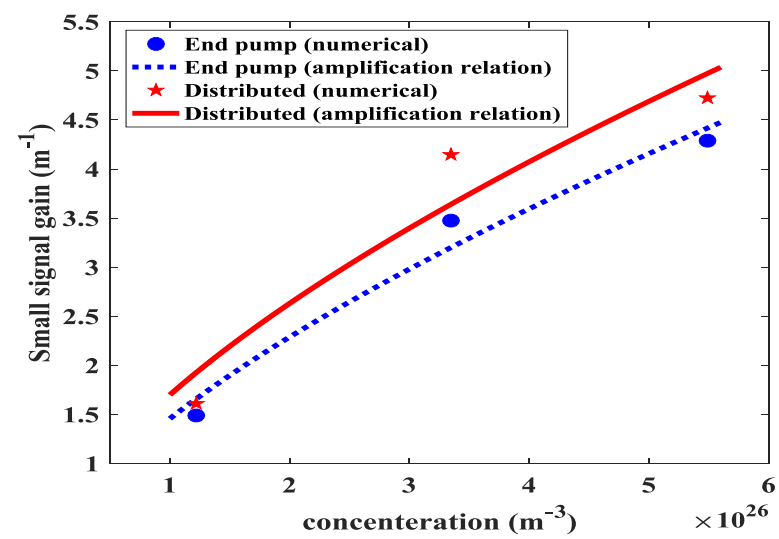

Fig. 7. Small-signal gain of end-pumping and distributed array versus concentration at a fixed 3.5 W pump power. 


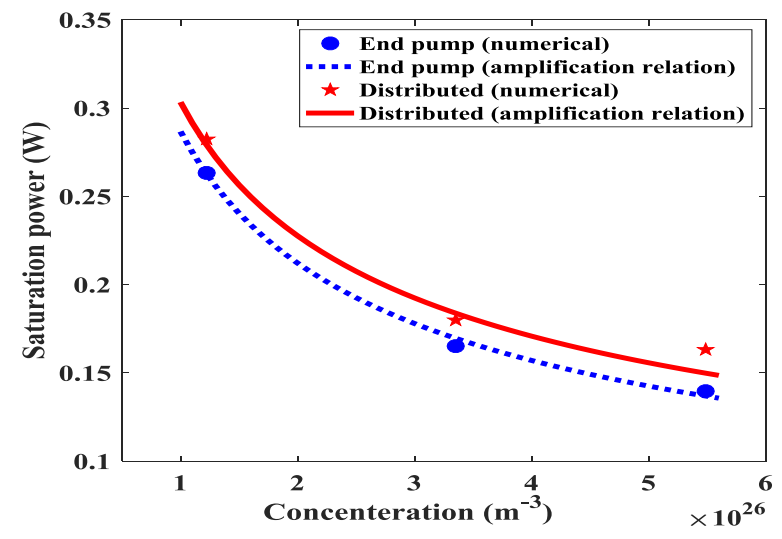

Fig. 8. Saturation power of end-pumping and distributed array versus concentration at a fixed 3.5 $\mathrm{W}$ pump power.

As a result, side-pumping is beneficial to improve the uniformity of the power distribution. The higher small-signal gain and the saturation power is obvious in both higher input pump powers and dopant concentrations for the distributed side-pumping array than end-pumping scheme. This is due to the more pumping points along the active fiber length for the distributed pumping technique in comparison to the end-pumping mode.

\section{CONCLUSION}

In this paper, the parameters of small-signal gain and saturation power of a typical Yb:silica DC QSW fiber amplifier are studied in various MOPA configurations such as; single-stage monolithic end-pumping and twostage distributed side-pumping modes. Furthermore, the dependence of cavity concentration and pump power on the amplification coefficients is investigated and analyzed. Numerical simulations have been performed using the time-dependent rate equations accompanying the transient amplification equation, in other to find the best values of $\gamma_{0}(z)$ and $P_{s a t}$, accordingly.

In fiber-based lasers, unlike other lasers, the amplification parameters are not constant, which is due to the DC nature of the optical fiber and the non-unformity of its injected pump power along the fiber length. The coefficients $\gamma_{0}(z)$ and $P_{\text {sat }}$ change linearly with increasing pump power, while their variations with fiber dopant concentration is exponential. It is notably due to the nonlinear nature of the population inversion and the pump/signal overlapping factors. The investigated parameters benefit higher values at distributed array than the end-pumping scheme, because of side-pumping occurs at $\mathrm{L} / 2$ in the distributed array.

The main advantage of this modeling (present work), arises from the nature of $\mathrm{z}$-dependent small-signal gain $\left(\gamma_{0}(z)\right)$. End pumping addresses inherently nonuniform excitation leading to $z$ dependent small-signal gain. However, according to the distributed amplification array, the side pumping allows us to improve the optical excitation along the fiber length leading to rather uniform smallsignal gain and the subsequent output power scale-up.

\section{REFERENCES}

[1] P. Parvin, M. Ilchi-Ghazaani, A. Bananej, and Z. Lali-Dastjerdi, "Small signal gain and saturation intensity of a $\mathrm{Yb}$ :Silica fiber MOPA system," Opt. Laser Technol. Vol. 41 pp. 885891, 2009.

[2] S. Mohammadian, P. Parvin, M. IlchiGhazaani, R. Poozesh, and K. Hejaz, "Measurement of gain and saturation parameters of a single-mode $\mathrm{Yb}$ : silica fiber amplifier," Opt. Fib. Technol. Vol. 19, pp. 446-455, 2013.

[3] J. Yoon-Chan, A. J. Boyland, J.K. Sahu, S.Chung, J. Nilsson, and D.N. Payne, "Multikilowatt single-mode ytterbium-doped largecore fiber laser," J. Opt. Soc. Korea, Vol. 13, pp. 416-422, 2009.

[4] M.J. Digonnet, Rare-earth-doped fiber lasers and amplifiers, revised and expanded, CRC Press, 2001.

[5] Z. Hanwei, X. Wang, P. Zhou, Z. Gong, and $\mathrm{X} . \mathrm{Xu}$, "6 $\mathrm{mJ}$, high-average-power, allfiberized Q-switched fiber master oscillator power amplifier with low repetition rate," Appl. Opt.. Vol. 51, pp. 6933-6936, 2012.

[6] W. Hanshuo, J. Song, J. Wu, J. Xu, H. Xiao, J. Leng, and P. Zhou, "High-power highly stable passively Q-switched fiber laser based on monolayer graphene," Laser Phys. Lett. Vol. 15, pp. 035102 (1-9), 2018. 
[7] B. Jaleh, P. Parvin, N. Sheikh, F. Ziaie, M. Haghshenas, and L. Bozorg, "Evaluation of physico-chemical properties of electron beamirradiated polycarbonate film," Radiat. Phys. Chem. vol. 76, pp. 1715-1719, 2007.

[8] S. Miclos, Savastru, D. Savastru, R. and Lancranjan, Numerical Simulation of Fiber Laser Operated in Passively Q-Switched and Mode-Locked Regimes. Fiber Laser, p. 275, 2016.

[9] Y. Wang, A. Martinez-Rios, and H. Po, "Analysis of a Q-switched ytterbium-doped double-clad fiber laser with simultaneous mode locking," Opt. Commun. Vol. 224, pp. 113-123, 2003.

[10] T. Challa, "Optimization of MOPA for YDDC Fiber Laser," Journal of Optoelectron. Eng. Vol. 2, pp. 29-32, 2014.

[11] J.A. Alvarez-Chavez, H.L. Of ferhaus, J. Nilsson, P.W. Turner, W.A. Clarkson, and D. J. Richardson, "High-energy, high-power ytterbium-doped Q-switched fiber laser," Opt. Lett.. Vol. 25, pp. 37-39, 2000.

[12] R. Čiegis, A. Dement'ev, and I. Laukaitytè, "A numerical algorithm for simulation of the Qswitched fiber laser using the travelling wave model," Lith. Math. J. Vol. 48, pp. 270-281, 2008.

[13] N. Shafii Mousavi, P. Parvin, and M. IlchiGhazaani, "Modeling of a Q-switched master oscillator power amplifier fiber laser and gain saturation properties," Laser Phys. Vol. 30, pp. 085101 (1-13), 2020.

[14] Y.E. Jeong, J.K. Sahu, D.A. Payne and J. Nilsson, "Ytterbium-doped large-core fiber laser with $1.36 \mathrm{~kW}$ continuous-wave output power," Opt. Express. Vol. 12, pp. 6088-6092, 2004.

[15] L. Jin, Y. Zou, X. Ma, Z.Yang, Z. Xue, Y. Jin, L. Xu, Q. Sui, W. Zhao, and Z. Zhang, "Simulation and optimization of pump technology on high-power fiber laser," International Conference on Optoelectronics and Microelectronics. IEEE, Vol. 6, pp. 77-80, 2012.

[16]H.G. Treusch, K. Du, M. Baumann, V. Sturm, B. Ehlers, and Loosen, "Fiber-coupling technique for high-power diode laser arrays. in Laser Resonators," International Society for Opt. Photon. Vol. 3267, pp. 0277 (1-9), 1998.
[17] L. Goldberg, B. Cole, and E. Snitzer, "Vgroove side-pumped $1.5 \mu \mathrm{m}$ fibre amplifier," Electron. Lett. Vol. 33, pp. 2127-2129, 1997.

[18] D. Ripin and L. Goldberg, "High efficiency side-coupling of light into optical fibres using imbedded v-grooves," Electron. Lett. Vol. 31, pp. 2204-2205, 1995.

[19] Y. Wang and C.-Q. Xu, "Modeling and optimization of Q-switched double-clad fiber lasers," Appl. Opt. Vol. 45, pp. 2058-2071, 2006.

[20] Y. Huo, R.T. Brown, G.G. King, and P.K. Cheo, "Kinetic modeling of Q-switched highpower ytterbium-doped fiber lasers," Appl. Opt. Vol. 43, pp. 1404-1411, 2004.

[21] L.M. Frantz and J.S. Nodvik, "Theory of pulse propagation in a laser amplifier," J. Appl. Phys. Vol. 34, pp. 2346-2349, 1963.

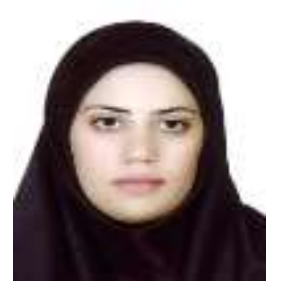

Narges Shafii Mousavi was born in Tehran, Iran, on July 7, 1983. She received the B.Sc. degree in physics from Islamic Azad University Science and Research Branch Tehran, Iran, in 2005, the M.Sc. Degree in optics \& Laser from Shiraz University, Shiraz, Iran, in 2009. She joined Amirkabir University for Ph.D. student, in 2013. Her current research interests include fiber grating, optical fiber, nonlinear optic, Q-switch fiber laser and Electromagnetically induced transparency.

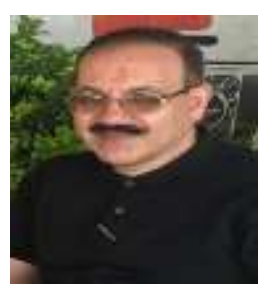

Parviz Parvin was born in Hamadan on 29 June 1962. Received the B.Sc. degree in electrical engineering from Shiraz University, 
Shiraz, Iran, in 1986, the M.Sc. degree in mechanical engineering from the Sharif Institute of Technology, Tehran, Iran, in 1989, and the Ph.D. degree in laser physics from the Amirkabir University of Technology (AUT), Tehran, in 1994. He has authored and coauthored more than 400 papers in refereed journals and international and domestic conferences, and holds four U.S. patents. He is currently a Professor with the Department of Energy Engineering and Physics and the Head of the Photonics Group at AUT. He has supervised more than 200 M.Sc. students and $30 \mathrm{Ph} . \mathrm{D}$. candidates. His research fields contain laser oscillator-amplifiers, IR/UV laser applications, IR/UV laser spectroscopy, lidars, remote sensing, nanostructure fabrication by lasers, bio photonics and fiber optics. He is currently involved in the entrepreneurship activities to commercialize the academic innovations through the establishment of knowledge-based companies. He has received the Sheikh Bahaii entrepreneurship award in 2012. Prof. Parvin is a Senior Member of the Optical Society of America, and a member of the European Optical Society and the Physics and Optics and Photonics Society of Iran.

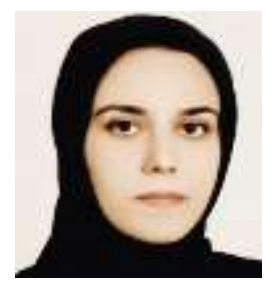

Maryam Ilchi-Ghazaani received the Ph.D. degree in laser physics from the Amirkabir University of Technology (AUT), Tehran, Iran. In 2015, she joined Nuclear Science and Technology Research Institute (NSTRI), as an assistant professor. She has been working on fiber lasers and amplifiers, laser fusion, laser accelerators and, laser cooling as well as the. Her current research focuses on laser medicine in the diagnosis and treatment of diseases. She is currently a juror of Elsevier, OSA journals $\&$ the Journal of Optoelectronical Nanostructures (JOPN). Dr. Ilchi has authored and co-authored several papers in refereed journals and international conferences, and holds two chapter books in InTechOpen entitled as "Fiber laser". 